\title{
A Novel Homozygous TTC7A Missense Mutation Results In Familial Multiple Intestinal Atresia And Combined Immunodeficiency
}

Wenjun Mou ( $\sim$ jenywenjun@126.com )

Beijing Children's Hospital Capital Medical University https://orcid.org/0000-0002-7781-7671

Shen Yang

Capital Medical University

Ruolan Guo

Beijing Pediatric Research Institute

Libing Fu

Capital Medical University

Li Zhang

East China Normal University

Weihong Guo

Capital Medical University

Jingbin Du

Capital Medical University

Jianxin He

Capital Medical University

Qinghua Ren

Capital Medical University

Yun Peng

Capital Medical University

Chanjuan Hao

Beijing Pediatric Research Institute

Jingang Gui

Capital Medical University https://orcid.org/0000-0003-3431-8749

Jinshi Huang

Capital Medical University

\section{Research Article}

Keywords: Tetratricopeptide repeat domain 7A, multiple intestinal atresia, neonatal sepsis, combined immunodeficiency 
Posted Date: July 8th, 2021

DOl: https://doi.org/10.21203/rs.3.rs-635099/v1

License: (c) (i) This work is licensed under a Creative Commons Attribution 4.0 International License. Read Full License

Version of Record: A version of this preprint was published at Frontiers in Immunology on December 15th, 2021. See the published version at https://doi.org/10.3389/fimmu.2021.759308. 


\section{Abstract}

Multiple intestinal atresia with combined immune deficiency (MIA-CID) are autosomal recessive disorders characterized by intestinal obstructions and profound immune defects. The study of patients with MIA and related disorders has established that tetratricopeptide repeat domain 7A (TTC7A) plays a critical role in intestinal and immune homeostasis and it is now shown that biallelic missense mutations have better survival outcomes. However, clues to related underlying molecular dysfunction remains elusive. In this study, we reported a patient with the diagnosis of severe CID and MIA that involved the pyloric diaphragm, ileum atresia, and colon stenosis, and the clinical course was complicated by multiple episodes of sepsis. In spite of multiple surgeries and supportive treatment, the patient died of severe sepsis and multiple organ failure at 3 months of age. The whole exome sequencing (WES) identified a novel homozygous TTC7A missense mutation (c.206T>C, p. L69P). The structural analysis showed that a hydrogen bond present between Gly65 and Leu69 in the wild-type TTC7A was broken by the Leu69Pro mutation. Moreover, this homozygous missense mutation led to a severely reduced TTC7A expression in lymphocytes and intestinal tissues, accompanied by prohibited lymphocyte development. Further studies demonstrated that the PI4K-FAM126A-EFR3A pathway was impaired in intestinal tissues. Our results strongly suggest that the missense mutation in TTC7A gene causes severe MIA-CID. More knowledge of the TTC7A protein functions will have important therapeutic implications for patients with MIA-CID.

\section{Introduction}

Multiple intestinal atresia (MIA) is an extremely rare and typically lethal congenital disorder involving multiple lesions at various levels throughout the intestine and colon. Associated combined immunodeficiency (CID) is also commonly present, allegedly caused by deficient thymopoiesis and lymphocyte differentiation, proliferation, and survival (1-5). Unlike classic CID in which opportunistic infections are common, patients with MIA-CID usually suffer from recurrent sepsis caused by intestinal bacteria $(6,7)$.

Recently, MIA-CID association was linked to mutations in tetratricopeptide repeat domain 7A (TTC7A) gene. TTC7A plays a vital role in epithelial cell growth, polarity, and differentiation in the gastrointestinal tract and thymus gland $(2,8)$. Since 2013 , there have been more than 50 patients with TTC7A mutations reported in the literature $(9,10)$. Loss-of-function mutations in TTC7A have been shown to cause immune and intestinal disorders of variable severity (11).

The composition of the plasma membrane in particular levels of phosphatidylinositol 4-phosphate (PI4P) are crucial for preserving epithelial cell polarity and survival. It has been confirmed that TTC7A acts as a scaffolding protein, where it binds and recruits phosphatidyl inositol 4 phosphatidylinositol 4-kinase III alpha (PI4KIIIa) to the cytoplasma membrane, facilitating the synthesis of PI4P $(1,10)$. TTC7A has also been reportedly interacts with the protein EFR3 homolog B (EFR3), which may serve as a membrane anchor for PI4K. Furthermore, TTC7A downregulates the RhoA-ROCK signaling pathway and the latter's downstream targets involved in actin dynamics (10). 
Although the causal nature of TTC7A to MIA-CID has been established through these findings and functional studies, questions remain on the exact correlation between mutations in this gene and phenotypic variations. The majority of the reported missense mutations are hypomorphic and had better survival outcomes. However, we report a patient of MIA-CID with a previously unreported homozygous missense mutation in TTC7A gene. This report adds to the ever-growing knowledge on MIA-CID that can contribute to the better understanding of these challenging disorders.

\section{Materials And Methods}

\section{Human sample collection}

Clinical information (including the demographic information, details of surgery, complications, clinical course, imaging examination results) and blood samples were collected from the patient, the parents, and controls, all of whom had given their prior informed consent to participation in the study. Genetic studies and data collection procedures involving human subjects conducted in the study were approved by the ethics committee of Beijing Children's Hospital. Ethics was approved by the Medical Ethical Committee of Beijing Children's Hospital which acted in compliance with ethical standards defined by the Declaration of Helsinki.

\section{Preparation of peripheral Blood Mononuclear Cells (PBMCs)}

Peripheral blood from the patient, the parents, and age- and sex-matched healthy controls were collected into EDTA-coated tubes. The PBMCs were isolated from peripheral blood by density gradient centrifugation using Ficoll-Hypaque. Briefly, $5 \mathrm{ml}$ freshly isolated EDTA anticoagulated blood was diluted with $5 \mathrm{ml} \mathrm{RPMI-1640} \mathrm{medium} \mathrm{(HyClone)} \mathrm{and} \mathrm{layered} \mathrm{onto} \mathrm{Ficoll-Hypaque} \mathrm{(Sigma)} \mathrm{in} \mathrm{a} \mathrm{3:1} \mathrm{ratio.} \mathrm{After}$ centrifuged at $1000 \mathrm{~g}$ for 20 minutes at room temperature, the buffy layer containing PBMCs was obtained, washed and re-suspended in RPMI-1640 medium supplemented with FBS and antibiotics cocktail (streptomycin and penicillin). Cell viability was determined by trypan-blue dye (Sigma) and cells were counted on an EVE ${ }^{\mathrm{TM}}$ automated cell counter (NanoEnTek).

\section{Histology and Immunohistochemistry}

Samples from biopsies were fixed in $10 \%$ buffered formalin, embedded in paraffin, and sections stained with hematoxylin and eosin according to standard protocols. Tissues from age-matched children were used as control tissues.

Immunohistochemistry was performed on $3 \mathrm{~mm}$ paraffine imbedded sections. Intestine sections were deparaffinized and rehydrated with successive baths of xylene and ethanol. Antigen retrieval was performed in $\mathrm{pH} 6.0$ citrate buffer for 20 minutes at $100^{\circ} \mathrm{C}$. Endogenous peroxidase activity was inhibited with $3 \%$ hydrogen peroxide ( $\mathrm{v} / \mathrm{v}$ in methanol). Sections were blocked for 30 minutes at room temperature in 1\% BSA PBS, then labeled using a rabbit anti-human TTC7A antibody (Proteintech, 21600-1-AP, 1:60 dilution) that recognized the sequence corresponding to amino acids $149-465$ at the $\mathrm{N}$-terminal of human 
TTC7A for 15 minutes. Anti-Rabbit HRP secondary antibody was applied at room temperature for 8 minutes. Signal amplification was performed using the DAB detection kit for 5 minutes and the cell nuclei were stained with hematoxylin. Sections were finally dehydrated and mounted, and then observed under a light microscope. Images were acquired with a bright-field light microscope at a magnification $\times 50$ to $\times 100$.

\section{Whole exome sequencing (WES) and variants analysis}

Genomic DNA was extracted from peripheral blood samples obtained from the proband and his parents using a QIAamp DNA blood midi kit (QIAGEN, Hilden, Germany) according to the manufacturer's protocol. Whole exome library was captured by a SureSelect Human All Exon Kit v6.0 (Agilent Technologies, Santa Clara, CA, USA). Targeted regions were sequenced on NovaSeq 6000 (Illumina, San Diego, USA) with $150 \mathrm{bp}$ pair-end runs. Sequencing reads were aligned to the GRCh37/hg19 human reference sequence. The mean coverage was $>100 \mathrm{X}$. Single nucleotide variants and indels were called using the SAMtools pipeline and annotated using ANNOVAR. Variant frequency was analyzed using 4 SNP databases (dbSNP 147, gnomAD, ExAC, and 1000 Genomes Project Database) and 2 disease databases (ClinVar and Human Gene Mutation Database). We filtered all common variants with minor allele frequency $>0.05$. For the candidate gene variants, we used the following 4 applications to predict their potential impact on protein function: SIFT (http://sift.jcvi.org/www/SIFT_enst_submit.html), Polyphen-2 (http://genet ics.bwh.harva rd.edu/pph2/), Mutation Taster (http://www.mutat ionta ster.org/), and CADD (http://cadd.gs.washi ngton.edu/). All candidate variants were classified and interpreted according to the American College of Medical Genetics and Genomics (ACMG) criteria (12). Putative pathogenic variants detected by WES were confirmed by Sanger sequencing. Primers were designed online using Primer 3.0.

\section{Conservation analysis of amino acid sequences of TTC7A}

Amino acid conservation was evaluated using the UGENE database with the sequences of Homo sapiens, Pan troglodytes, Macaca mulatta, Bos taurus, Mus musculus, Rattus norvegicus, Gallus gallus, Danio rerio and Xenopus (Silurana) tropicalis.

\section{Structural analysis of TTC7A}

The sequences of TTC7A were obtained from the UniProtKB (UniProtKB: Q9ULT0). The crystal structure of TTC7A (SMTL ID: 5dse.2.A) was used as the template, which was determined by X-ray diffraction at a resolution of $2.9 \AA$. The amino acid residue at coordinate 69 was mutated for variant Leu69Pro. The structures were analyzed with PyMOL software. The residue 69, together with certain nearby ones within $3 \AA$, was illustrated. For clear demonstration of interresidue relationship, some residues were highlighted in the indicated colors with the computed hydrogen bonds being labeled.

\section{Western blot analysis}


Total protein was extracted from PBMCs of the patient, and age- and sex-matched healthy controls using RIPA buffer supplemented with $100 \mathrm{mM}$ PMSF and complete protease inhibitor cocktail (Roche Molecular Biochemicals). Prepared samples were electrophoresed in SDS-PAGE gels following protein quantitation by BCA protein assay (Pierce, Thermo fisher scientific) according to the manufacturer's protocol and equivalent protein amounts were loaded. Proteins were then blotted onto PVDF membranes. The membranes were probed with appropriate primary and secondary antibodies, developed using ECL substrate, and images were acquired using a chemiluminescence imaging system. The following primary antibodies were used: TTC7A (Proteintech, 21600-1-AP, 1:300 dilution), b-actin (Cell Signaling, 4970, 1:2000 dilution).

\section{q-PCR analysis}

Total RNA was extracted from intestinal specimens by using a RNeasy Kit (Qiagen, Hilden, Germany). Quantitative PCR (qPCR) was performed in an ABI Prism 7300 sequence detecting system (Applied Biosystems). A list of primers used is included in Table 1.

\section{Table 1. Primer list and probe sequences}

\begin{tabular}{ll} 
Primer & Sequence (5'-3') \\
\hline 18S rRNA-F & GTAACCCGTTGAACCCCATT \\
\hline 18S rRNA-R & CCATCCAATCGGTAGTAGCG \\
\hline TTC7A-F & GCACCTCAAGGAAGCAGGTT \\
\hline TTC7A-R & GCTATGCATGATGCGCACG \\
\hline PI4KA-F & CCATGAAGTGGGCACCTACC \\
\hline PI4KA-R & CCATACACCCCAAGAGTTGTG \\
\hline PI4KB-F & CCCCCAGAGCCTGTTCGAC \\
\hline PI4KB-R & AGTGGGCAGCCAGACTCG \\
\hline FAM126A-F & GTTGTGGAGGAATGGTTGTCA \\
\hline FAM126A-R & GACAGGTTCTAGCAACTCACTT \\
\hline FAM126B-F & CAGAGTAATGGTTGCATTGAAGC \\
\hline FAM126B-R & AGGGGATAGTGAAAGACAGAACT \\
\hline EFR3A-F & GGATCGAATTGGTTCTTACCTGG \\
\hline EFR3A-R & GGCTTAATGCTTTGAGAATGGCA \\
\hline EFR3B-F & GGTACGTGTGCATTGCTATGG \\
\hline EFR3B-R & TGGCAAACTTCACAAACGAGT
\end{tabular}




\section{Flow cytometry}

Red blood cells-removed peripheral blood was incubated with the fluorochrome-conjugated monoclonal antibodies for 20 minutes at room temperature in the presence of Fc blocking (BD Biosciences) reagents. All the antibodies used were from BD Pharmingen (BD Biosciences, US) and listed below: CD3 (OKT3), CD19 (4G7), CD4 (OKT4), CD8 (RPA-T8), CD45RA (HI100), CD24 (ML5), CD38 (HIT2), CD16 (3G8), and CD56 (B159). Samples were acquired on a BD LSRFortessa flow cytometer (BD Biosciences), with FlowJo (Tree Star, Ashland, Oregon, USA) as the analysis software.

\section{RNA extraction, sequencing and analysis}

Total RNAs were extracted from blood samples and purified Oligo (dT) beads. The RNA libraries were constructed by poly $(A)$ protocol. Fragmentation was performed using Fragmentation Buffer. First-strand cDNA was synthesized using a random hexamer primer and M-MuLV Reverse Transcriptase (RNase H Minus). Second-strand cDNA synthesis was subsequently performed using DNA polymerase I and RNase $\mathrm{H}$. The cDNA libraries were sequenced by Illumina X-ten platform using paired-end $150 \mathrm{bp}$ reads.

The paired-end reads were mapped to human reference genome and Ensembl gene annotation (GRCh37/hg19 assembly) using hisat v2.2.1 (13). The mapped reads were then processed by Stringtie v2.1.4 to quantitate the gene expression against the Ensembl transcript annotation (14). The gene expression data was normalized to fragments per kilobase of transcript per million mapped reads (FPKM). The differentially expressed genes (DEGs) were identified based on the fold change between the proband and his parents (fold change $>2$ or $<0.5$ ). The gene set enrichment analysis (GSEA) was conducted to identify the Gene Ontology (GO) terms or pathways overrepresented by the DEGs. The pathways used for GSEA were curated by KEGG (Kyoto Encyclopedia of Genes and Genomes) (15), Reactome (16), and WikiPathway (17), and collected from MSigDB database. The GSEA was implemented by $\mathrm{R}$ enricher function in clusterProfiler package (18).

\section{Statistical analysis}

Differences between groups were calculated using unpaired Student $t$-test with GraphPad Prism 9. Data were presented as the mean \pm standard error of the mean (SEM). Statistical significance was defined as *, $P<0.05 ; \star \star, P<0.01 ; * \star \star, P<0.001$.

\section{Results}

\section{Identification and intestinal histopathology of the MIA patient}

The proband was a boy born to 3-generation consanguineous parents of Chinese origin, as shown in Fig. 1A. The proband was the fifth of 5 children of the couple. The first child of the parents was diagnosed with MIA and died in the first month of life because of post-operation complications consisting of severe septicemia and multiple organ failure. The second and fourth child could not eat after birth and died in the first months of life without going to the hospital. The third child was 11 years old and asymptomatic. 
His mother also had a history of previous abortion (fifth pregnancy, Fig. 1A). The proband's uncle (father's younger brother) has congenital absence of the left forearm, and other relatives did not have other congenital malformations and diseases.

The proband was delivered by vaginal delivery at 34 weeks estimated gestational age. At birth, neonatal intestinal perforation and meconium peritonitis secondary to intestinal obstruction was suspected. The patient was transferred to our hospital and underwent surgery on his third day, during which the ileum atresia, and pyloric diaphragm were removed, and an ileum ostomy was performed because of distal colon obstruction. The proband subsequently suffered from severe sepsis and persistent watery diarrhea (through the distal ileum ostomy) with no relief to aggressive antibiotic and parenteral nutrition. The proband underwent multiple surgeries and was finally given a diagnosis of MIA that involved the pyloric diaphragm, ileum atresia, and colon stenosis.

During hospitalization, the patient's clinical course was complicated by multiple episodes of sepsis. The patient had seven episodes of hyperpyrexia associated with elevated C-reactive protein (CRP), white blood cell (WBC) and neutrophils. Klebsiella oxytoca and Methicillin-Resistant Staphylococcus epidermidis were detected by separate bilateral blood culture. Recurrent and refractory sepsis was diagnosed with poor respond to antibotics of Aztreonam, Amikacin, Meropenem, Fluconazole. In spite of three times of surgeries and supportive treatment, the patient died of severe sepsis and multiple organ failure at 3 months of age.

In Fig. 1B, pathology results of the ileum atresia showed mucosal erosion, epithelial depolarized, diffused apoptotic-like changes, and dense mixed inflammatory infiltrated within the lamina propria with a majority of lymphocytes. Inherent glands are dilated and twisted, with diffused submucosal patchy lymphoid hyperplasia.

\section{A novel homozygous missense mutation was identified in exon 2 of TTC7A gene through WES}

To dissect the genetic cause that could possibly associate with the disease, we carried out the WES using the patient's and his parents' genomic DNA. We identified a novel homozygous missense mutation c.206T > C in exon 2 of TTC7A gene in the patient that had not been reported in the Clinvar and the Human Gene Mutation Database (HGMD). The unaffected parents were heterozygous for TTC7A missense mutation c.206T >C. Sanger sequencing of TTC7A gene confirmed this variant of the patient and suggested one allele was inherited from his father, and the other from mother (Fig. 1C). Missense mutation pathogenicity prediction was performed using MutationTaster software and the results showed that the mutation was predicted to be damaging.

Phylogenetic analysis indicated that the position surrounding the proline 69 residue were highly conserved across different species by UGENE software, Fig. 2A. As shown in Fig. 2B, this site-mutation could cause a leucine to proline change at position 69 of the peptide which located in exon 2 ( $p$. Leu69Pro). Structural analysis showed that the hydrogen bond present between Gly65 and Leu69 in the wild-type TTC7A was broken by the Leu69Pro mutation, Fig. 2C. 
Taken together, we concluded that the homozygous missense mutation c.206T > C was the causal mutation for the patient.

\section{The homozygous TTC7A missense mutation leads to a $\mathrm{T}^{\text {low }} \mathrm{B}^{-} \mathrm{NK}^{\text {low }}$ immunotype}

In a subset of TTC7A mutated patients, MIA is associated with CID (3). Thus, we examined whether the immunotype of the patient was affected. Immunologic profiling of the patient's PBMCs revealed significantly low T (both CD4 and CD8) and NK cells (CD3, 184 cells/ $\mu$ l, reference 2179-4424 cells/ $\mu$; CD4, 47 cells/ $\mu \mathrm{l}$, reference 1461-3018 cells/ $\mu$ l; CD8, 123 cells/ $\mu$, reference 556-1687 cells/ $\mu$ l; NK cells, 155 cells/ $\mu$ l, reference $290-780$ cells/ $\mu l$, Table 2). In addition, majority of T cells in the patient's CD4 and CD8 T cells were of memory phenotype without CD45RA expression, Table 2. The patient showed markedly elevated double negative $\mathrm{T}\left(\mathrm{DNT}, \mathrm{CD} 3^{+} \mathrm{CD} 4^{-} \mathrm{CD} 8^{-}\right.$) cells, which suggested that $\mathrm{T}$ cell differentiation might be changed, Fig. 3A. Taken together, these results indicated that the homozygous TTC7A missense mutation led to an abnormal T-cell and NK-cell development.

Table 2

Immunological phenotypes of the TTC7A patient.

\begin{tabular}{|c|c|c|c|c|}
\hline & \multicolumn{2}{|l|}{ Patient } & \multicolumn{2}{|c|}{ Control value range } \\
\hline & $\%$ & Absolute (cells/ $\mu \mathrm{l})$ & $\%$ & Absolute (cells/ $\mu \mathrm{l})$ \\
\hline \multicolumn{5}{|l|}{ T cells } \\
\hline CD3+ & $6.09 \%$ & 184 & $54.28-71.67^{a}$ & $2179-4424^{a}$ \\
\hline $\mathrm{CD} 3+\mathrm{CD} 4+$ & $1.54 \%$ & 47 & $33.72-52.43^{a}$ & $1461-3018^{a}$ \\
\hline $\mathrm{CD} 3+\mathrm{CD} 8+$ & $4.06 \%$ & 123 & $14.08-24.70^{a}$ & $556-1687^{a}$ \\
\hline CD $4+$ CD 45RA+ & $6.87 \%$ & 13 & $69.15-88.10^{a}$ & $1170-2595^{a}$ \\
\hline CD8 + CD45RA+ & $16.61 \%$ & 31 & $68.90-94.60^{a}$ & $503-1276^{a}$ \\
\hline \multicolumn{5}{|l|}{ B cells } \\
\hline CD19+ & $0.53 \%$ & 16 & $17.34-36.03^{a}$ & $734-2265^{a}$ \\
\hline \multicolumn{5}{|l|}{ NK cells } \\
\hline CD3-CD16 + CD56+ & $5.13 \%$ & 155 & $5.89-14.85^{a}$ & $290-780^{a}$ \\
\hline a $1-6$ months (1). & & & & \\
\hline
\end{tabular}

\section{Reference:}

1. Ding Y, Zhou L, Xia Y, Wang W, Wang Y, Li L, et al. Reference values for peripheral blood lymphocyte subsets of healthy children in China. J Allergy Clin Immunol. 2018;142(3):970-3 e8. 
The phenotype of B cells was also analyzed. The patient exhibited a significantly decreased number of peripheral B cells ( 16 cells $/ \mu l$, reference $734-2265$ cells $/ \mu l$, Table 2$)$. The proportion of CD19 ${ }^{+} B$ cells in lymphocytes were markedly reduced $(0.5 \%$, reference $17.3-36.0 \%$, Table 2$)$. We then analyzed the subpopulation of B cells. The B cells were predominantly transitional B cells (CD24 ${ }^{\text {bright }}$ CD38 $8^{\text {bright }}$ ) and virtually no $C D 24^{+} C D 38^{\text {dim }}$ naive $B$ cells or $C D 24^{+} C D 38^{-}$memory $B$ cells were shown in the patients (naive $B$ cell, $60.5 \%$ versus $11.4 \%$; memory $B$ cells, $14.2 \%$ versus $1.49 \%$; transitional $B$ cells, $13.6 \%$ versus $77.2 \%$, Fig. 3B). Taken together, these results indicated that the homozygous TTC7A missense mutation led to a compromised B-cell development.

Collectively, these results demonstrated that the homozygous TTC7A missense mutation led to a compromised lymphocyte development.

\section{The homozygous TTC7A missense mutation leads to diminished TTC7A expression}

We speculated if this novel homozygous missense mutation might affect the TTC7A expression. We found that patient's PBMCs expressed very low level of TTC7A compared with healthy controls by Western blot, Fig. 4A. We then tested the TTC7A expression in mRNA level in patient's intestinal tissue, qPCR analysis indicated that the expression level of TTC7A was lower in the proband compared with patients with Hirschsprung's disease (HD), Fig. 4B. Immunohistochemical staining of patient's iluem tissue confirmed a reduced level of TTC7A expression compared with the meconium ileus patient, Fig. 4C. Taken together, these results indicated that this homozygous TTC7A missense mutation could lead to reduced TTC7A expression in lymphocytes and intestinal tissues.

\section{Loss of TTC7A expression results in a prohibited lymphocyte development}

To shed light on the functional impact of TTC7A variants on the gene expression, we compared the gene expression profile of the patient with those of his parents, and identified a total of 4,852 differentially expressed genes (1,826 upregulated and 3,026 downregulated) between the proband and the parents (fold change $>2$ or $<0.5$ ).

Subsequently, the upregulated and downregulated genes were subjected to GSEA analysis, respectively. Worth to be noted, the downregulated genes were significantly enriched in $\mathrm{GO}$ terms and pathways related to $\mathrm{T}$ - and B-cell proliferation, differentiation and activation, and NK-cell mediated cytotoxicity (Fig. $5 A, B)$, which might account for the $T^{\text {low }} B^{-} N^{l o w}$ immunotype observed. Taken together, these results demonstrated that the loss of TTC7A of the current patient resulted in a prohibited lymphocyte development.

\section{Loss of TTC7A expression results in impaired PI4K-FAM126A-EFR3A pathway in intestinal tissues}

Avitzur et al. have shown that TTC7A mutations directly reduced the transport of PI4KIIIa, a major TTC7Ainteracting protein via coimmunoprecipitation experiments, into plasma membrane for membranous stabilization (1). We next examined the expression of PI4KA and PI4KB through qPCR experiments in 
intestinal tissue and found that $P / 4 K A$ and $P I 4 K B$ expression were significantly reduced in patient's intestinal tissue compared with that of patients with HD, Fig. 6A, 6B. As the TTC7A-PI4K complex is stabilized by an adaptor protein, FAM126A, and once at the plasma membrane, tethered by EFR3, a membrane-bound protein $(10,19)$. We next confirmed $F A M 126 A / B$ expression through qPCR experiments in intestinal tissue of the patient. The results showed that the intestinal tissue of patient had possessed much lower levels of FAM126A and FAM126B mRNA compared with that of patients with HD, Fig. 6C, 6D. We also observed reduced expression levels of EFR3A in the patients' intestinal tissue, Fig. 6E. However, the intestinal tissue of patient showed comparable EFR3B expression compared with that of patients with HD, Fig. 6F.

Previous studies reported that TTC7A protein was supposed to act as a repressor of RhoA signaling pathway, crucial to normal ROCK function $(4,7)$. As shown in Fig. 6G, 6H, reduced levels of ROCK1 and $R O C K 2$ expression were detected in the intestinal tissue between the patients and controls.

Taken together, these results indicated that loss of TTC7A of the current patient resulted in the dysregulation of the PI4K-FAM126A-EFR3A pathway, rather than the ROCK pathway, in intestinal tissues.

\section{Discussion}

TTC7A deficiency is an autosomal-recessively inherited disease. Multiple mutations in the TTC7A gene, combined with heterogenous disease presentation involving inflammatory, gastrointestinal and immunological manifestations (10), has made it difficult to correlate phenotype and genotype (20). In this study, we presented a new case of familial MIA-CID with a novel homozygous TTC7A missense mutation.

There exists a strong correlation between genetic mutations and the survival of patients and their clinical manifestations. The majority of the reported missense mutations were hypomorphic and had better survival outcomes. These patients were likely to have missense mutations without TPR domain involvement, which mediating protein-protein interaction. Under these circumstances, it is possible that residue function of the protein may be retained, which may correspond to a relatively better chance of survival. The patient in current study died even after aggressive treatment, unlike most other patients with TTC7A missense mutations. This might be related to the missense mutation he carried, and resulted in disruption of these TPR domains. The reduced TTC7A expression was also consistent with this. The diminished TTC7A expression in PBMCs and intestinal tissues of the current patient, as confirmed by western blot, qPCR and immunohistochemical experiments, suggested the loss of TTC7A protein function, consistent with the severity of the clinical manifestations.

Less than $20 \%$ of TTC7A-deficiency patients presents with MIA alone (20). The incidence of MIA accompanying CID is higher, approximately $75 \%$, in TTC7A-deficiency patients $(5,20)$. Intestinal disease manifestations can present as early in utero, whereas CID may be difficult to recognize before birth (10). The patient in current study had severe T and B cell and mild NK cell lymphopenia, which might be caused by a prohibited lymphocyte development. Indeed, our GSEA analysis showed that the downregulated genes were significantly enriched in GO terms and pathways related to lymphocyte 
development. Besides, the results of this patient's chest X-ray examination showed that the thymus was smaller than aged-matched healthy control. Accordingly, our GSEA analysis also showed that the downregulated genes were significantly enriched in GO terms and pathways related to T cell differentiation in thymus. These results indicated the abnormalities in thymus development. However, it is still unclear whether the T cell immunodeficiency of the disease results primarily from defects of thymic epithelial cells, or from abnormalities intrinsic to the T cell lineage, or both.

Here we show that the homozygous TTC7A missense mutation c.206T > C impairs the PI4KA-FAM126AEFR3A signaling pathway in a way that impairs lymphocyte homeostasis (Supplementary Fig. 1) and intestinal epithelial depolarization. TTC7A is known to regulate cell adhesion, polarization, and migration by inducing the phosphorylation of ROCK effectors. Avitzur et al. have shown impaired cell adhesion to collagen and fibronectin in TTC7A-depleted cells (1). Accordingly, our GSEA analysis showed that the downregulated genes were also significantly enriched in $\mathrm{GO}$ terms and pathways related to cell adhesion.

Unfortunately, there is no standard therapy for TTC7A-deficiency and traditional therapies do little to treat the intestinal disease (MIA and very early onset inflammatory bowel disease, VEOIBD). Former studies showed that surgical resections for MIA did not prevent the formation of new atresias (21). Intestinal epithelial cell apoptosis and chronic diarrhea are refractory to traditional IBD therapies including immunosuppressive treatments $(1,22)$. Although many new treatment strategies, including early total enterectomy combined with parenteral nutrition, prophylaxis immunoglobulin, antibiotic, and small-bowelliver transplant, have been reported successfully, their safety and limitations remain to be evaluated in the future $(23,24)$. Hematopoietic stem cell transplantation (HSCT) has been reported to restore immunologic defects to overcome thymus dysfunction, however, the epithelial phenotype is only marginally attenuated, suggesting that intestinal disease is independent of the immune deficiency $(9,20)$. Also, early recognition of the immunodeficiency and early intervention with IgG replacement and prophylactic antimicrobials may provide time to better assess and monitor the immune system in order to make long-term therapeutic decisions for these patients.

The PI4KA-TTC7A-EFR3 or ROCK-TTC7A signaling pathways may become therapeutic targets. TTC7A protein, through defective control of RhoA signaling pathway activation, interferes with key cellular functions in epithelial and lymphocyte homeostasis (10). The administration of ROCK inhibitors is thought to ameliorate proliferative activity and epithelial architecture of the lumen and intestinal crypts. In particular, disturbed intestinal epithelia and abnormalities of thymic thymocytes associated with excessive RhoA signaling can be reversed in 3D organoids cultures in vitro by treatment of Y-27632 (2). This opens the perspective of future therapeutic option based on use of ROCK inhibitors.

In conclusion, TTC7A mutations should be investigated in patients with MIA with or without CID. Our study expands the spectrum of TTC7A mutations in MIA-CID. Characterization of the role of TTC7A protein in the immune system and intestinal development, as well as in thymic epithelial cells, might have important therapeutic implications in MIA-CID patients in the future. On the other hand, further studies are 
needed to determine and recognize whether modified mechanisms can compensate defective TTC7A signaling to open alternative therapeutic avenues.

\section{Declarations}

\section{Funding}

This work was supported by grants from National Natural Science Foundation of China (No. 81802491).

\section{Conflicts of interest}

The authors declare that they have no conflict of interest.

\section{Availability of data and materials}

Not applicable.

\section{Code availability}

Not applicable.

\section{Authors' contributions}

WM, SY, JG and SJH designed most of the studies. WM and SY carried out much of the work together with RG, LF, LZ, QR, WG, JD, CH and YP. SY, WM, XJH, JG and SJH analyzed the data. WM wrote up the manuscript with input from SY, JG, JH, LZ and RG. All authors read and approved the final manuscript.

\section{Ethics Approval}

The study was approved by the Ethics Committee of Beijing Children's Hospital, Capital Medical University, and performed according to the Declaration of Helsinki. The written informed consents for publication were obtained from the parents of the patient.

\section{Consent to participate}

Clinical information and blood samples were collected from the patient, the parents, and controls, all of whom had given their prior informed consent to participation in the study.

\section{Consent for publication}

All authors consent to publish the manuscript.

\section{References}


1. Avitzur Y, Guo C, Mastropaolo LA, Bahrami E, Chen H, Zhao Z, et al. Mutations in tetratricopeptide repeat domain 7A result in a severe form of very early onset inflammatory bowel disease. Gastroenterology. 2014;146(4):1028-39.

2. Bigorgne AE, Farin HF, Lemoine R, Mahlaoui N, Lambert N, Gil M, et al. TTC7A mutations disrupt intestinal epithelial apicobasal polarity. J Clin Invest. 2014;124(1):328-37.

3. Chen R, Giliani S, Lanzi G, Mias GI, Lonardi S, Dobbs K, et al. Whole-exome sequencing identifies tetratricopeptide repeat domain 7A (TTC7A) mutations for combined immunodeficiency with intestinal atresias. J Allergy Clin Immunol. 2013;132(3):656-64. e17.

4. Lemoine R, Pachlopnik-Schmid J, Farin HF, Bigorgne A, Debre M, Sepulveda F, et al. Immune deficiency-related enteropathy-lymphocytopenia-alopecia syndrome results from tetratricopeptide repeat domain 7A deficiency. J Allergy Clin Immunol. 2014;134(6):1354-64. e6.

5. Mandia N, Perez-Munuzuri A, Lopez-Suarez O, Lopez-Sanguos C, Bautista-Casanovas A, Couce ML. Congenital intestinal atresias with multiple episodes of sepsis: A case report and review of literature. Med (Baltim). 2018;97(23):e10939.

6. Yang W, Lee PP, Thong MK, Ramanujam TM, Shanmugam A, Koh MT, et al. Compound heterozygous mutations in TTC7A cause familial multiple intestinal atresias and severe combined immunodeficiency. Clin Genet. 2015;88(6):542-9.

7. Notarangelo LD. Multiple intestinal atresia with combined immune deficiency. Curr Opin Pediatr. 2014;26(6):690-6.

8. Fernandez I, Patey N, Marchand V, Birlea M, Maranda B, Haddad E, et al. Multiple intestinal atresia with combined immune deficiency related to TTC7A defect is a multiorgan pathology: study of a French-Canadian-based cohort. Med (Baltim). 2014;93(29):e327.

9. Kammermeier J, Lucchini G, Pai SY, Worth A, Rampling D, Amrolia P, et al. Stem cell transplantation for tetratricopeptide repeat domain 7A deficiency: long-term follow-up. Blood. 2016;128(9):1306-8.

10. Jardine S, Dhingani N, Muise AM. TTC7A: Steward of Intestinal Health. Cell Mol Gastroenterol Hepatol. 2019;7(3):555-70.

11. El-Daher MT, Lemale J, Bruneau J, Leveau C, Guerin F, Lambert N, et al. Chronic Intestinal PseudoObstruction and Lymphoproliferative Syndrome as a Novel Phenotype Associated With Tetratricopeptide Repeat Domain 7A Deficiency. Front Immunol. 2019;10:2592.

12. Richards S, Aziz N, Bale S, Bick D, Das S, Gastier-Foster J, et al. Standards and guidelines for the interpretation of sequence variants: a joint consensus recommendation of the American College of Medical Genetics and Genomics and the Association for Molecular Pathology. Genet Med. 2015;17(5):405-24.

13. Kim D, Langmead B, Salzberg SL. HISAT: a fast spliced aligner with low memory requirements. Nat Methods. 2015;12(4):357-60.

14. Pertea M, Pertea GM, Antonescu CM, Chang TC, Mendell JT, Salzberg SL. StringTie enables improved reconstruction of a transcriptome from RNA-seq reads. Nat Biotechnol. 2015;33(3):290-5. 
15. Kanehisa M, Furumichi M, Sato $Y$, Ishiguro-Watanabe M, Tanabe M. KEGG: integrating viruses and cellular organisms. Nucleic Acids Res. 2021;49(D1):D545-D51.

16. Croft D, O'Kelly G, Wu G, Haw R, Gillespie M, Matthews L, et al. Reactome: a database of reactions, pathways and biological processes. Nucleic Acids Res. 2011;39(Database issue):D691-7.

17. Slenter DN, Kutmon M, Hanspers K, Riutta A, Windsor J, Nunes N, et al. WikiPathways: a multifaceted pathway database bridging metabolomics to other omics research. Nucleic Acids Res. 2018;46(D1):D661-D7.

18. Yu G, Wang LG, Han Y, He QY. clusterProfiler: an R package for comparing biological themes among gene clusters. OMICS. 2012;16(5):284-7.

19. Baskin JM, Wu X, Christiano R, Oh MS, Schauder CM, Gazzerro E, et al. The leukodystrophy protein FAM126A (hyccin) regulates Ptdlns(4)P synthesis at the plasma membrane. Nat Cell Biol. 2016;18(1):132-8.

20. Lien R, Lin YF, Lai MW, Weng HY, Wu RC, Jaing TH, et al. Novel Mutations of the Tetratricopeptide Repeat Domain 7A Gene and Phenotype/Genotype Comparison. Front Immunol. 2017;8:1066.

21. Ali YA, Rahman S, Bhat V, Al Thani S, Ismail A, Bassiouny I. Hereditary multiple intestinal atresia (HMIA) with severe combined immunodeficiency (SCID): a case report of two siblings and review of the literature on MIA, HMIA and HMIA with immunodeficiency over the last 50 years. BMJ Case Rep. $2011 ; 2011$.

22. Thiagarajah JR, Kamin DS, Acra S, Goldsmith JD, Roland JT, Lencer WI, et al. Advances in Evaluation of Chronic Diarrhea in Infants. Gastroenterology. 2018;154(8):2045-59 e6.

23. Fayard J, Collardeau S, Bertrand Y, Cordier MP, Malcus C, Dubois R, et al. TTC7A mutation must be considered in patients with repeated intestinal atresia associated with early inflammatory bowel disease: Two new case reports and a literature review. Arch Pediatr. 2018.

24. Gilroy RK, Coccia PF, Talmadge JE, Hatcher LI, Pirruccello SJ, Shaw BW Jr, et al. Donor immune reconstitution after liver-small bowel transplantation for multiple intestinal atresia with immunodeficiency. Blood. 2004;103(3):1171-4.

\section{Figures}


A

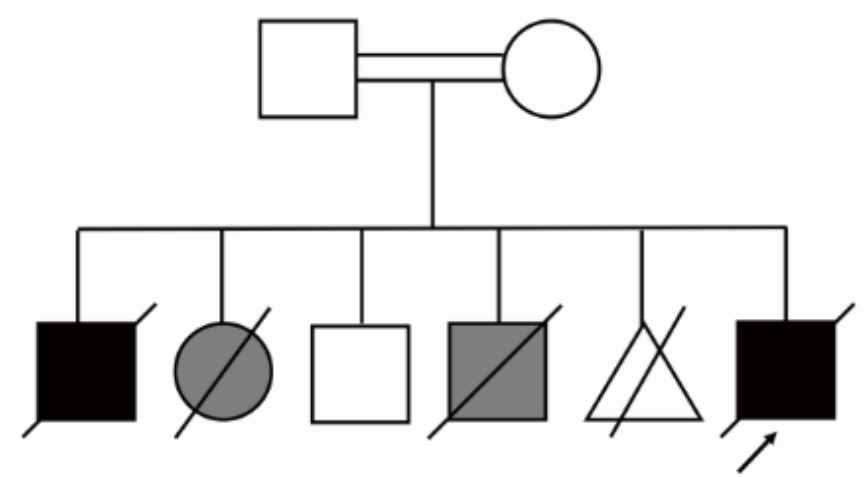

B

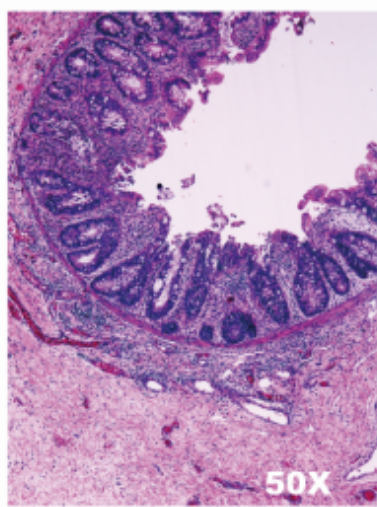

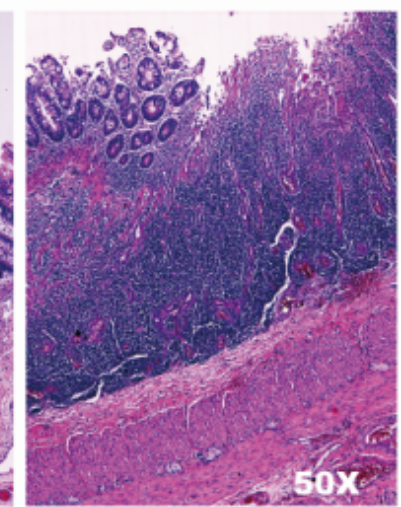

C
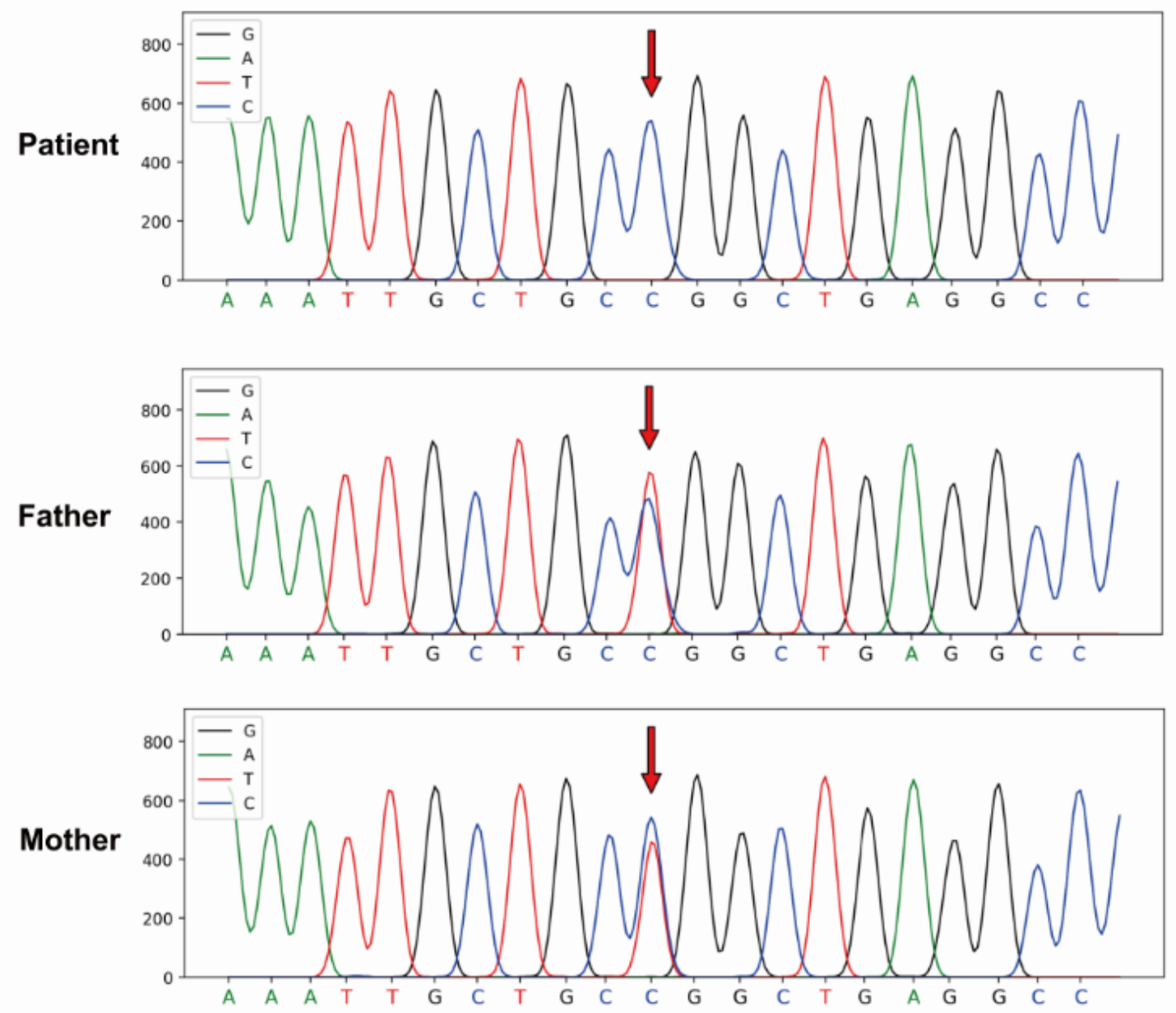

Figure 1

A novel homozygous TTC7A missense mutation was identified in the patient with familial MIA. A. Pedigree chart of the proband's family. Consanguinity was indicated with a double horizontal line. Males were depicted as squares, females as circles, decreased individuals with a diagonal line. Abortion was depicted as triangle with a diagonal line. Black filled symbols represented individuals who were clinically affected, while grey filled symbols indicated individuals who did not receive medical testing. The proband who underwent WES were indicated by an arrow. B. H\&E photomicrographs of patient's small bowel of 
these atretic areas showed mucosal erosion, diffused apoptotic-like changes, and dense mixed inflammatory infiltrate within the lamina propria with marked eosinophilia. C. Sanger sequencing chromatogram depicted the 206T>C missense mutation of TTC7A.

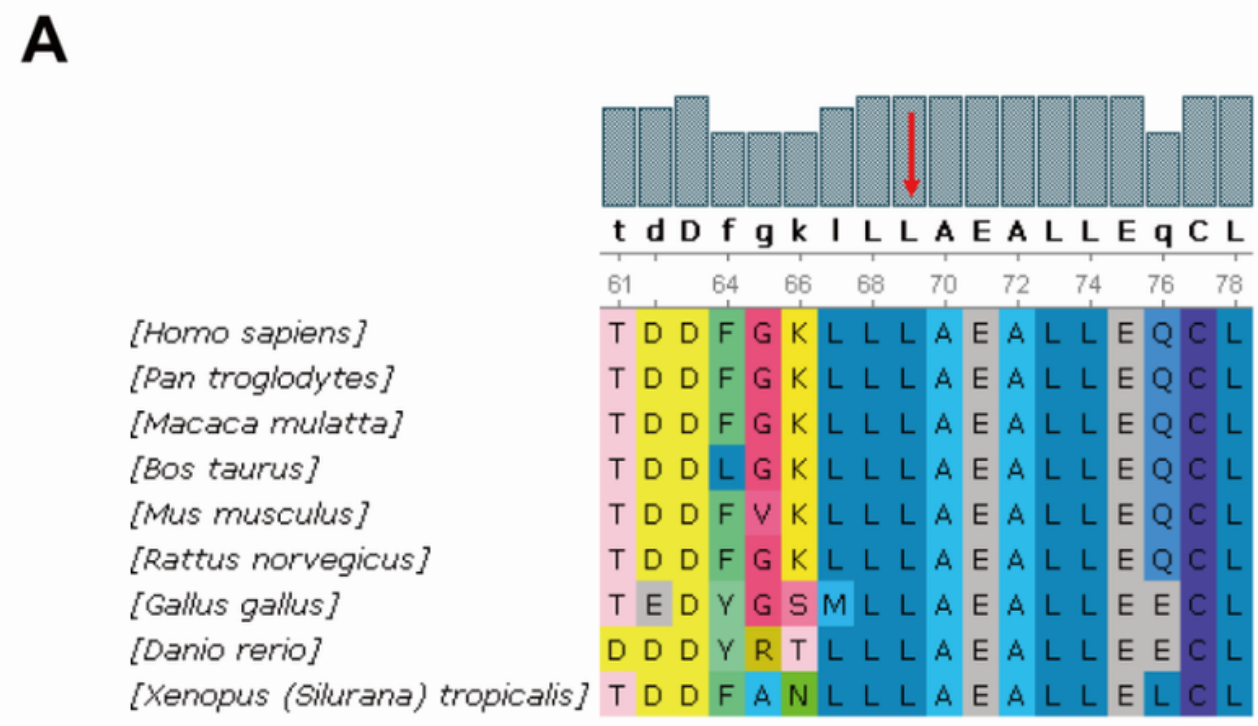

B

c.206 T>C, p. L69P

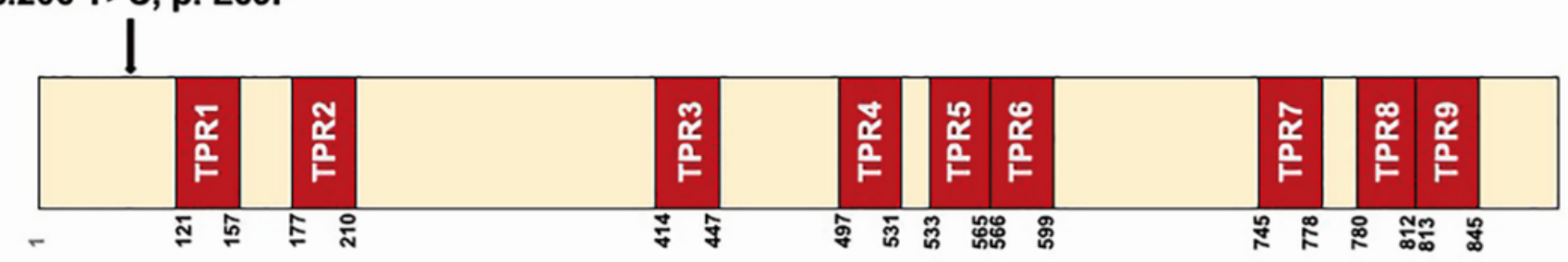

C

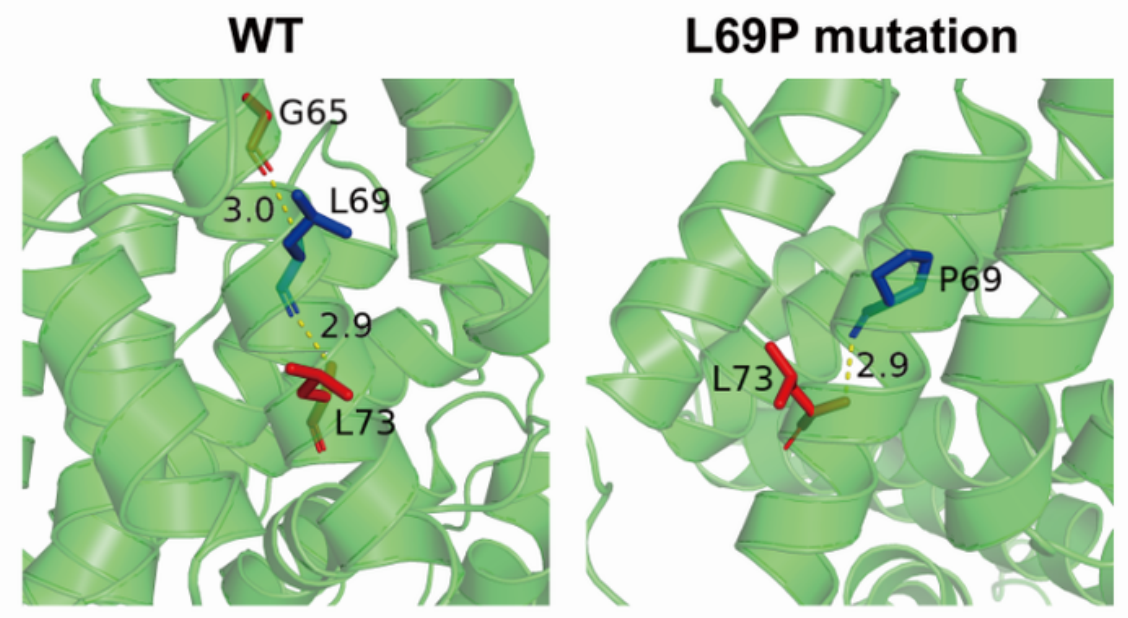

Figure 2

Structure analysis of the mutant TTC7A and Amino acid conservation for the p. L69P variants. A. Protein alignment indicated that the position of L69P mutation was highly conserved across different species. Residue L69 was shown in red arrow. B. The schematic structure of TTC7A showed the location of the 
variant identified in this study. Illustration of TTC7A protein with TPR domains was in red and the identified mutation were highlighted. C. The hydrogen bond presenting between Gly65 and Leu69 in the wild-type TTC7A was broken by the L69P mutation. The residue 69 together with certain nearby residues within $3 \AA$ was illustrated in the wild-type (WT) and the mutant by PyMOL. Computed hydrogen bonds were shown as yellow dashed lines. Residues Leu69/Pro69 were highlighted in blue.
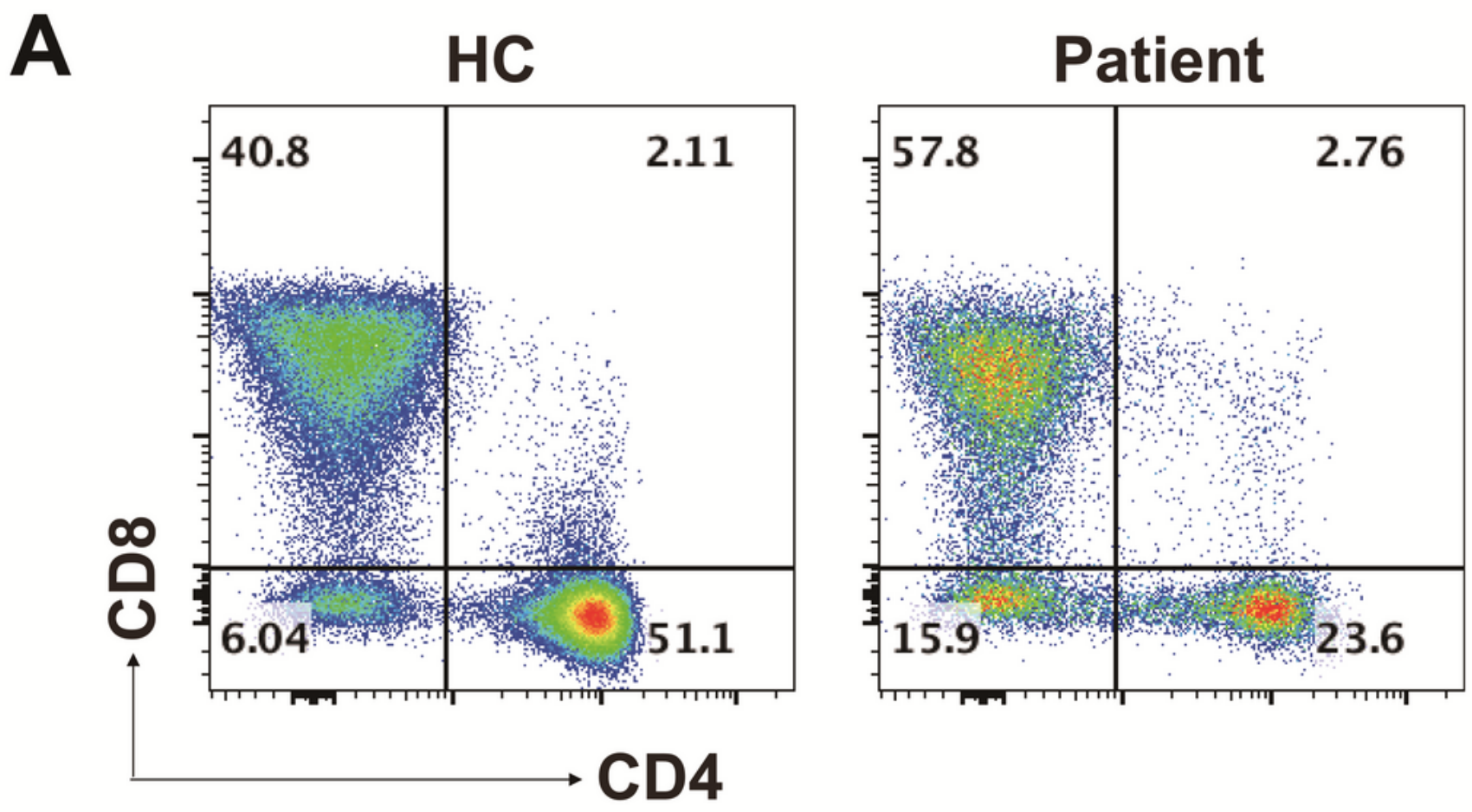

B

\section{HC}

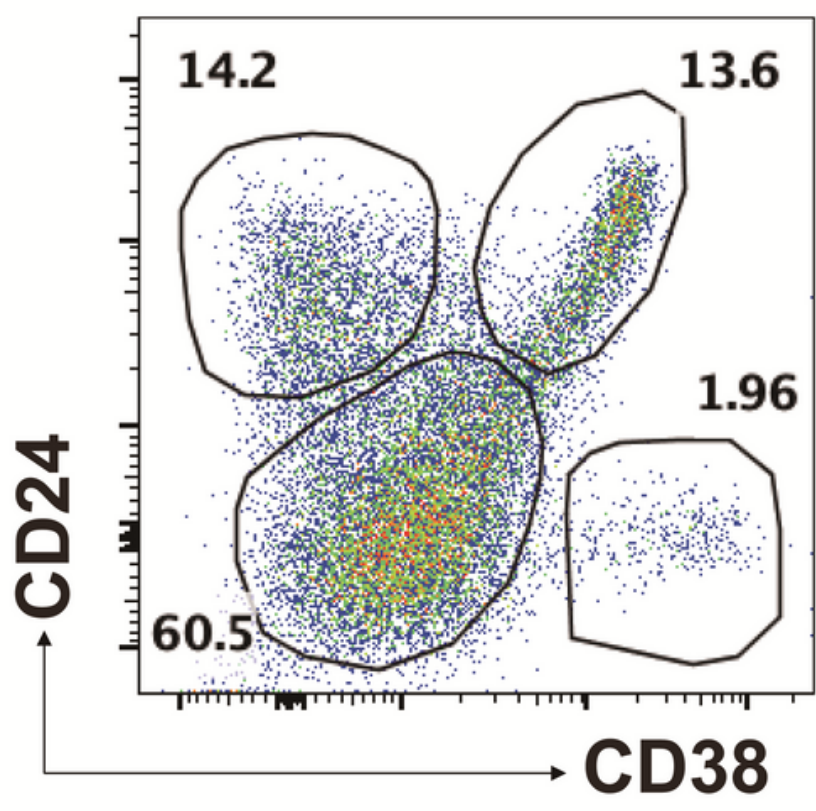

Patient

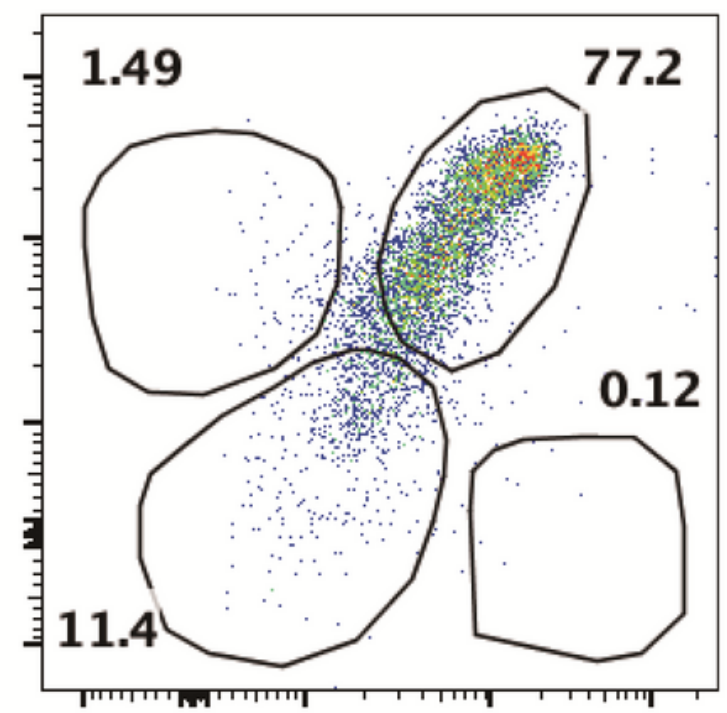

Figure 3 
Increased frequencies of DNT and transitional B cells. A. Representative dot plots showed markedly elevated frequencies of DNT compared with age- and sex-matched healthy control (HC), as measured by flow cytometry. B. Representative dot plots showed significantly increased frequencies of CD24brightCD38bright transitional B cells, as measured by flow cytometry.

A

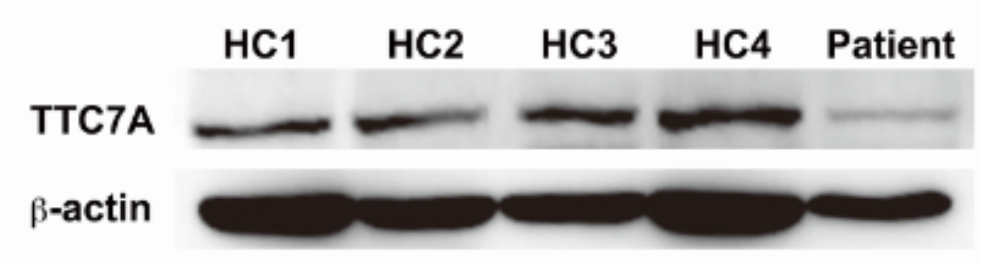

C

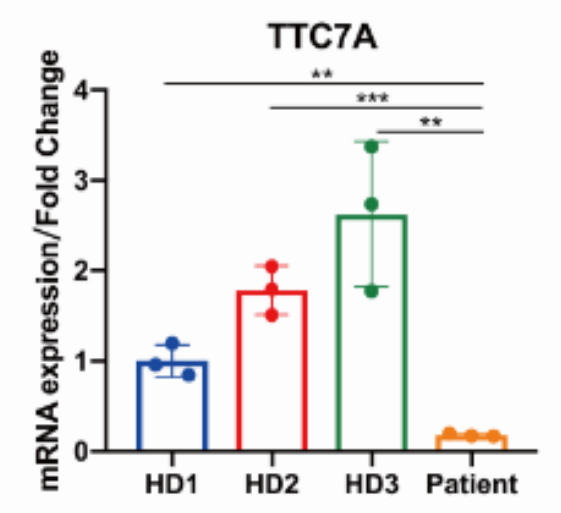

D

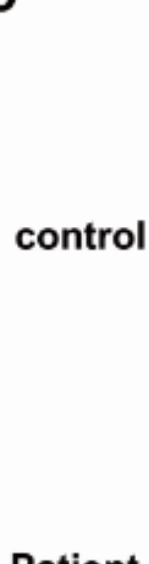

B

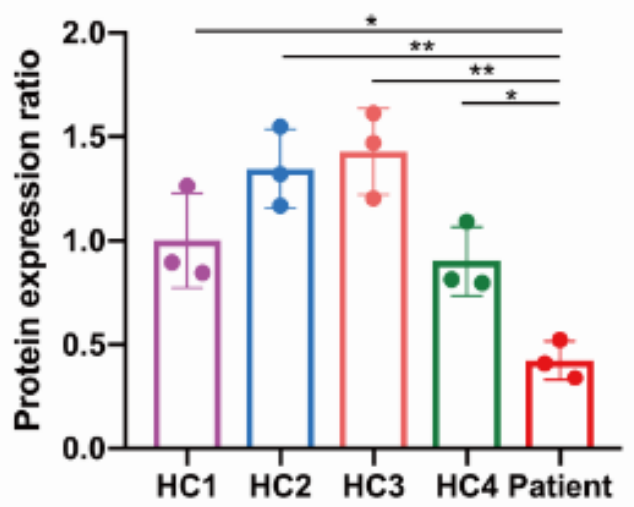

$50 x$
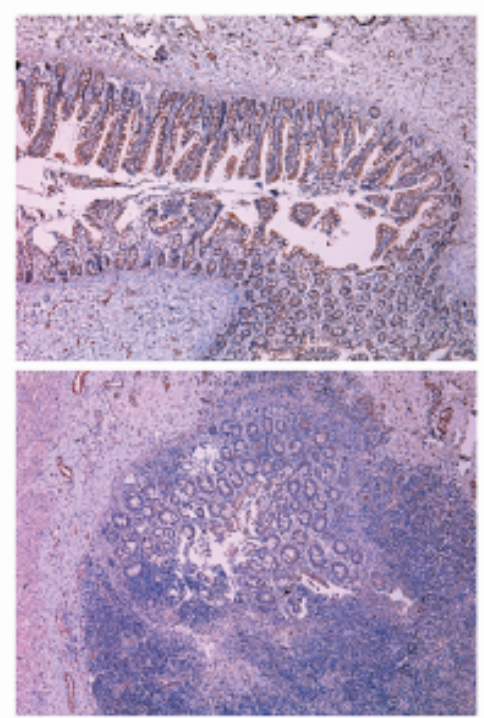

$100 x$

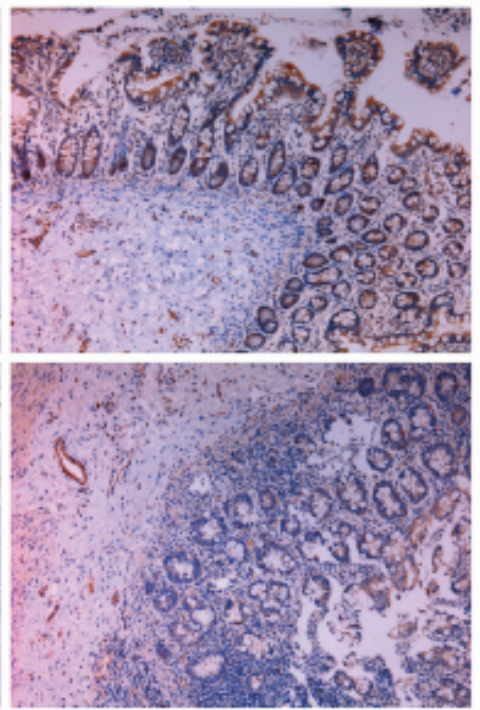

\section{Figure 4}

Diminished TTC7A expression in lymphocytes and intestinal tissues. A. Western blot of patient-derived PBMCs showed a severely impairment of TTC7A protein expression compared with healthy controls (HC). $\beta$-actin was used as an internal loading control in the western blot. The result was a representative of 3 independent experiments. B. The protein band intensity was quantified and analyzed by Image J software. C. The qPCR results demonstrated that patient's intestinal tissue had a significant reduction in the amount of TTC7A expression compared with Hirschsprung's disease (HD). TTC7A expression was normalized to $18 \mathrm{~S}$ rRNA, and results were represented as relative expression normalized to the control. $\star \star P<0.01,{ }^{\star \star *} P<0.001$. D. In the patient's intestinal tissue, immunohistochemistry demonstrated that TTC7A protein expression was diminished compared with the meconium ileus patient (control). Magnification for left panel was $\times 50$, magnification for right panel was $\times 100$. 
A

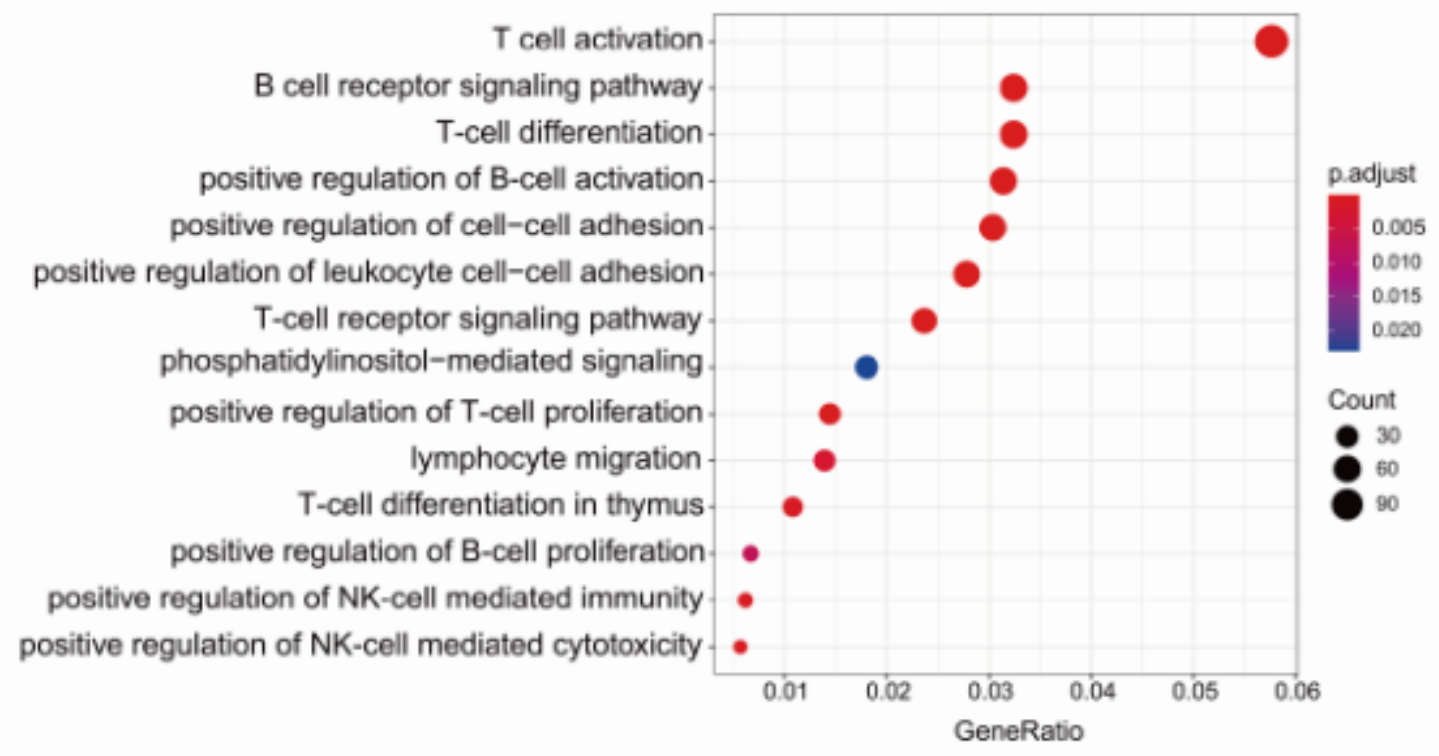

B

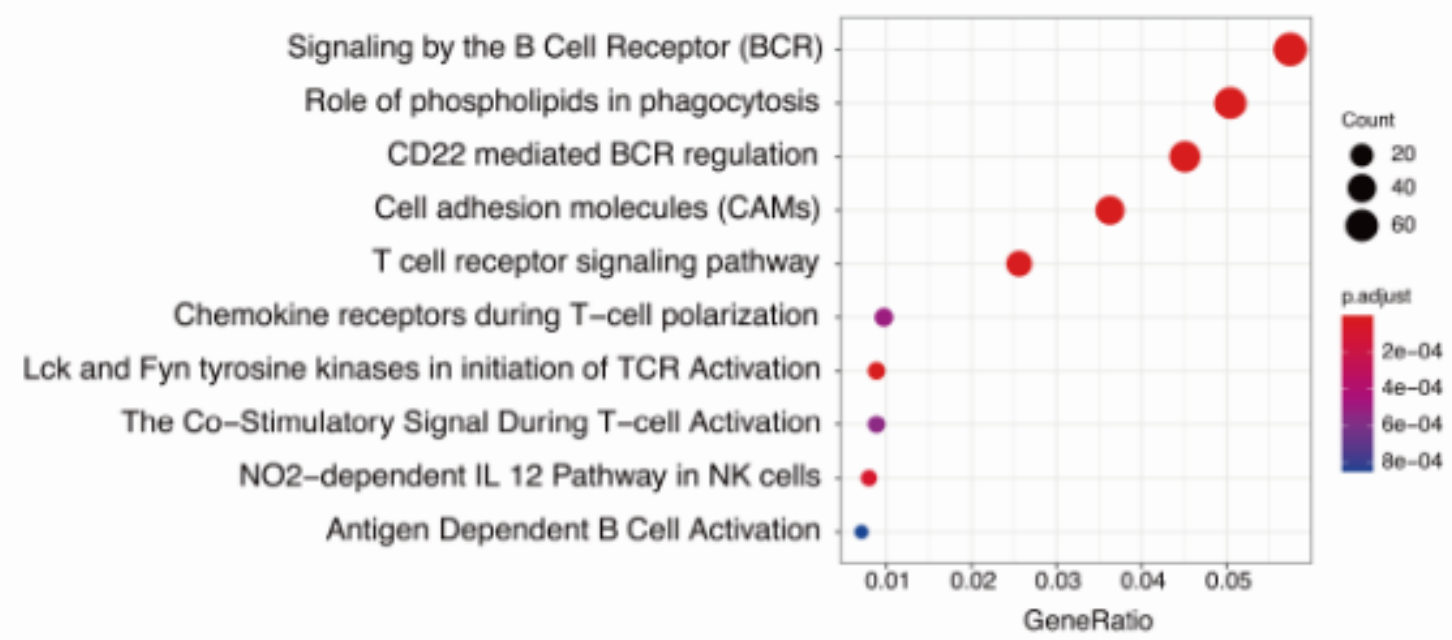

\section{Figure 5}

GO and pathway analysis indicated a prohibited lymphocyte development and function. GO terms (A) and pathways $(B)$ enriched by the downregulated genes in the patient. Enriched pathways with an adjusted $P$ value of less than 0.05 were considered significantly enriched. 
A

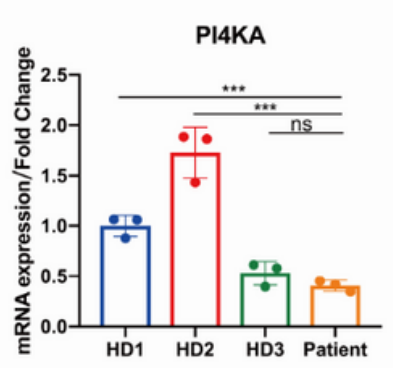

E

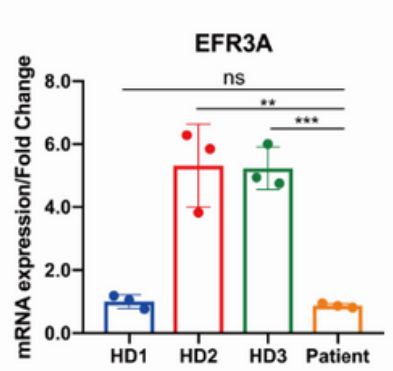

B

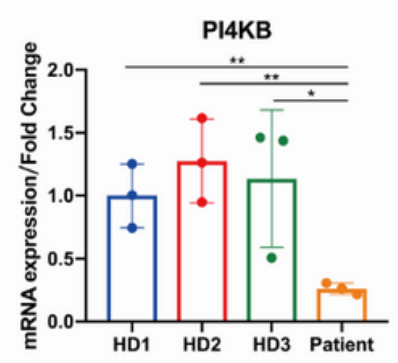

$\mathbf{F}$

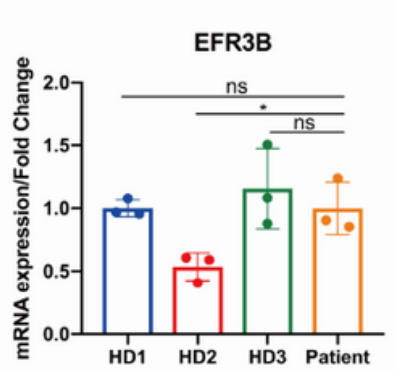

C

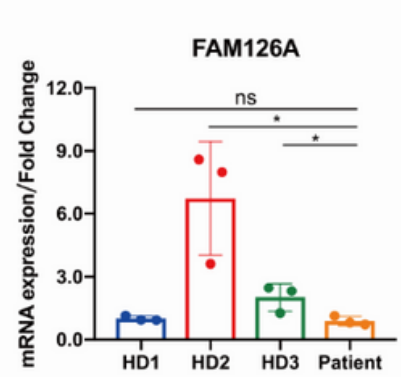

G

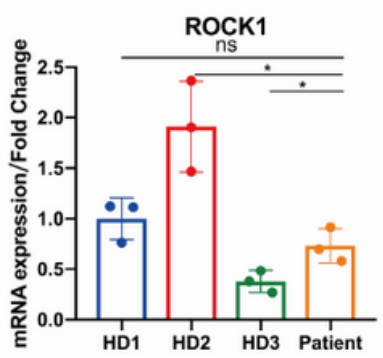

D

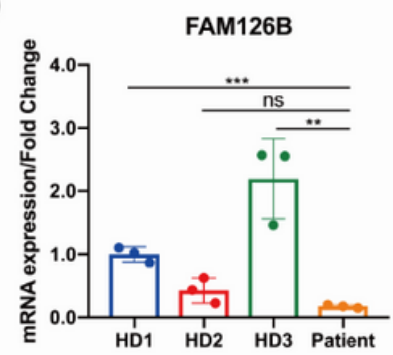

H

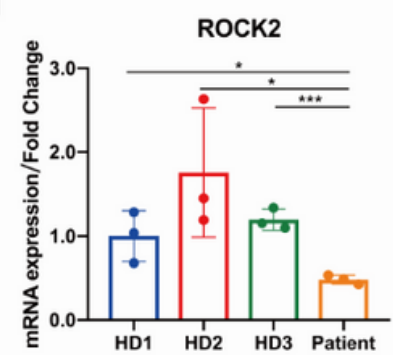

Figure 6

Impaired PI4K-FAM126A-EFR3A pathway in patient's intestinal tissues. (A-B). qPCR analysis of patientderived intestinal biopsy specimens showed reduced PI4KA and PI4KB expression compared with Hirschsprung's disease (HD). (C-E, G, H). qPCR analysis of patient-derived intestinal biopsy specimens showed reduced FAM126A (C), FAM126B (D), EFR3A (E), ROCK1 (G), and ROCK2 (H) expression compared with HD. (F). qPCR analysis of patient-derived intestinal biopsy specimens showed comparable EFR3B expression compared with HD.

\section{Supplementary Files}

This is a list of supplementary files associated with this preprint. Click to download.

- SupplementaryFigure1.tif 